\title{
Strain-related regional alterations of calcium-handling proteins in myocardial remodeling
}

\author{
Ahmet Kilic, MD, ${ }^{a}$ Tieluo Li, MD, ${ }^{a}$ Timothy D. C. Nolan, MS, ${ }^{\text {a }}$ Jennifer R. Nash, MS, ${ }^{\text {a }}$ Shuying Li, MD, \\ Deyanira J. Prastein, MD, ${ }^{a}$ Gary Schwartzbauer, PhD, ${ }^{a}$ Sina L. Moainie, MD, ${ }^{a}$ G. Kwame Yankey, MD, ${ }^{a}$ \\ Christopher DeFilippi, MD, ${ }^{\mathrm{b}}$ Zhongjun $\mathrm{Wu}, \mathrm{PhD},{ }^{\mathrm{a}}$ and Bartley P. Griffith, $\mathrm{MD}^{\mathrm{a}}$
}

From the Division of Cardiac Surgery, ${ }^{\mathrm{a}} \mathrm{De}-$ partment of Surgery, and Division of Cardiology, ${ }^{\mathrm{b}}$ Department of Medicine, University of Maryland School of Medicine, Baltimore, Md.

Read at the Eighty-sixth Annual Meeting of The American Association for Thoracic Surgery, Philadelphia, Pa, April 29-May 3, 2006.

The study is partially supported by a William G. McGowan Charitable Fund grant, National Institutes of Health grant R01EB02076, and National Institutes of Health HL072751 training grant (A.K. and G.K.Y.).

Received for publication May 5, 2006; revisions received June 14, 2006; accepted for publication July 7, 2006.

Address for reprints: Bartley P. Griffith, MD, University of Maryland School of Medicine, Department of Cardiothoracic Surgery, 22 S. Greene St, UMMS, N4W94, Baltimore, MD 21201 (E-mail: bgriffith@ smail.umaryland.edu).

J Thorac Cardiovasc Surg 2006;132:900-8

$0022-5223 / \$ 32.00$

Copyright (๑) 2006 by The American Association for Thoracic Surgery

doi:10.1016/j.jtcvs.2006.07.016
Background: Cardiac remodeling has been shown to have deleterious effects at both the global and local levels. The objective of this study is to investigate the role of strain in the initiation of structural and functional changes of myocardial tissue and its relation to alteration of calcium-handling proteins during cardiac remodeling after myocardial infarction.

Methods: Sixteen sonomicrometry transducers were placed in the left ventricular free wall of 9 sheep to measure the regional strain in the infarct, adjacent, and remote myocardial regions. Hemodynamic, echocardiographic, and sonomicrometry data were collected before myocardial infarction, after infarction, and 2, 6, and 8 weeks after infarction. Regional myocardial tissues were collected for calciumhandling proteins at the end study.

Results: At time of termination, end-systolic strains in 3 regionally distinct zones (remote, adjacent, and infarct) of myocardium were measured to be $-14.65 \pm 1.13$, $-5.11 \pm 0.60(P \leq .05)$, and $0.92 \pm 0.56(P \leq .05)$, respectively. The regional end-systolic strain correlated strongly with the abundance of 2 major calciumhandling proteins: sarcoplasmic reticulum $\mathrm{Ca}^{2+}$ adenosine triphosphatase subtype $2 \mathrm{a}\left(r^{2}=0.68, P \leq .05\right)$ and phospholamban $\left(r^{2}=0.50, P \leq .05\right)$. A lesser degree of correlation was observed between the systolic strain and the abundance of sodium/calcium exchanger type 1 protein $\left(r^{2}=0.17, P \leq .05\right)$.

Conclusions: Regional strain differences can be defined in the different myocardial regions during postinfarction cardiac remodeling. These differences in regional strain drive regionally distinct alterations in calcium-handling protein expression.

A fter amyocardial infarction (MI), the heart undergoes alterations at the myocyte level that lead to changes in global function, known as remodeling. ${ }^{1}$ The International Forum on Cardiac Remodeling defines the change as, "genome expression, molecular, cellular and interstitial changes that are manifested clinically as changes in size, shape, and function of the heart after cardiac injury." Although initially an adaptive and compensatory mechanism, progressive cardiac remodeling has a deleterious and negative impact.

Gradients of electromechanical function and associated protein expression occur among 3 histologically different zones. ${ }^{3-5}$ The 3 distinct zones include (1) the relatively normal healthy myocardium (remote zone), (2) the nonischemic but hypokinetic area near the MI (adjacent zone), and finally (3) the area of fibrosis and scar (infarct zone). The loss of mechanical function and its associated gene expression of calcium handling in the infarct zone is due to ischemic necrosis; however, the differences between the nonischemic adjacent and remote zones are less easily understood. We have been interested in the regional differences in post-MI strain that might explain alterations in regional function through mechanotransduction. 


\section{Abbreviations and Acronyms \\ $\mathrm{LV}=$ left ventricular \\ MI = myocardial infarction \\ NCX-1 = sodium/calcium exchanger type 1 \\ PLB $=$ phospholamban \\ SERCA2a $=$ sarcoplasmic reticulum $\mathrm{Ca}^{2+}$ adenosine triphosphatase subtype $2 \mathrm{a}$}

\section{Methods}

\section{Surgical Protocol}

Twelve Dorsett hybrid sheep between 50 and $70 \mathrm{~kg}$ and bred for laboratory use (Thomas Morris, Reisterstown, Md) were used in the study. Nine animals were instrumented with subsequent creation of an anterior MI. The sheep were allowed to recover and survived for 8 to 12 weeks after the initial MI. Three noninstrumented animals were used for healthy tissue controls. All the animals received treatment in compliance with the "Guide for the Care and Use of Laboratory Animals" published by the National Institutes of Health (National Institutes of Health publication 8523 , revised 1985). The surgical procedures and postoperative care were carried out according to the approved protocol by the Institutional Animal Care and Use Committee of the University of Maryland at Baltimore.

Anesthesia was induced by thiopental sodium $(10 \mathrm{mg} / \mathrm{kg})$ and maintained by $1 \%$ to $2 \%$ isoflurane (Draeger anesthesia monitor, North American Draeger, Telford, Pa). Surface electrocardiogram, arterial blood pressure, pulse oximeter, and esophageal temperature were continuously monitored for each animal during the operations described. The instrumented group underwent a left anterolateral thoracotomy with excision of the left fifth rib. Polypropylene snares were placed around the first and second diagonal coronary arteries of the left anterior descending artery and passed through pressure tubing. The snares were momentarily tightened $(<30$ seconds) to demarcate the border of the future infarct. Four specific transducers ( $2 \mathrm{~mm}$; Sonometrics Corporation, London, Ontario, Canada) were placed at the superior, inferior, medial, and lateral aspects of the transiently ischemic myocardium with an additional transducer placed in the center of this ischemic region. An additional 11 transducers were sutured into the midmyocardium of the left ventricular (LV) free wall to create a final array of 3 short-axis aligned rows of 5 transducers with an additional transducer in the apex. The wires of the transducers were secured together with silk ties, tunneled subcutaneously, and their respective skin buttons exposed to allow for future data acquisition. The coronary snares were tunneled subcutaneously for subsequent permanent vessel occlusion. An ultrasonic flow probe (20 $\mathrm{mm}$; Transonic Systems, Inc, Ithaca, NY) was placed around the main pulmonary artery for cardiac output monitoring. An atrial port silicone catheter (9F; Access Technologies, Stokie, Ill) was placed into the left atrium and placed in a subcutaneous pocket for future myocardial perfusion measurements.

\section{Infarction}

Seven to 10 days later, the sheep were reanesthetized and a catheter-tip mounted pressure transducer (SPC 350; Millar Instru- ments, Inc, Houston, Tex) was placed by fluoroscopy into the LV apex via the femoral artery. A midline laparotomy was made for subdiaphragmatic echocardiographic imaging. After all preinfarction baseline data (sonomicrometry, echocardiogram, hemodynamics) were recorded, the subcutaneous snares were permanently tightened to cause an anterior MI and the animal was supported with epinephrine infusion. The epinephrine infusion $(240 \mu \mathrm{g} / \mathrm{h})$ was started at the time of initial snare occlusion and lasted for 15 minutes, at which point the ionotrope was serially weaned in increments of $60 \mu \mathrm{g} / \mathrm{h}$ every 5 minutes until the animal was off epinephrine support. The induction of MI was seen as electrocardiographic changes initially and later confirmed by echocardiography. After an additional 15 minutes off epinephrine support, all immediate post-MI data were collected. The midline incision was closed and the animal was allowed to recover.

\section{Data Collection}

Transdiaphragmatic echocardiograms with sonomicrometry and hemodynamic data were collected at the time of infarction (before and after MI), 2 weeks and 6 weeks after MI, and at the time of terminal study. Echocardiograms were collected with a Sonos 5500 machine with a sterile covered transducer (Philips Medical, Andover, Mass). Sonomicrometry data were collected with a commercially available digital sonomicrometry system (Sonometrics Corporation). The pulmonary artery flow rate was measured with a transonic flowmeter (T401; Transonic Systems). Distance between all pairs of 16 transducers (120 unique distances) was measured at a sampling rate of 200 samples/sec, in real time, and synchronized with LV pressure and pulmonary artery flow.

\section{Data Analysis}

The LV short-axis views at the tips of the papillary muscles, at the base of the papillary muscles, and at the apex along with long-axis views were studied. LV volumes and infarct size expressed as percentage of endocardial circumference were measured and the ejection fraction was calculated by the Bullet formula. ${ }^{6}$

By use of the signal post-processing software and multidimensional scaling algorithm available from Sonometrics (Sonoview and Sonoxyz), the distances between the implanted 16 sonomicrometry transducers were first filtered to remove noises, and the instantaneous location of each transducer in a single 3-dimensional coordinate system was determined. The coordinate data were used to determine 3-dimensional motion and deformation of the LV free wall. The arrangement of the transducers consisted of 3 groups of 5 placed circumferentially along the LV free wall, with 1 transducer being placed into the LV apex.

The strain measure during an individual cardiac cycle is often referred to as the systolic strain whereas the strain measure used over time is referred to as the remodeling strain. ${ }^{7,8}$ For the present study, an area strain measure was used and calculated by comparing the area change of the paired triangles between the reference frame and the deformed frames. Strain measurements were then calculated from the collected sonomicrometry transducer coordinate data to compile (1) an end-systolic regional strain and (2) an end-diastolic (remodeling) strain over the progression of myocardial remodeling.

The end-systolic regional strain was calculated with the LV free wall deformation during an individual cardiac cycle to 
TABLE 1. Summary of echocardiographic data

\begin{tabular}{llccrr}
\hline Study time points & LVESV $(\mathbf{m L})$ & LVEDV $(\mathbf{m L})$ & EF (\%) & $\begin{array}{c}\text { WMA length } \\
\text { (cm) }\end{array}$ & WMA/Circ \\
\hline Preinfarct & $33.54 \pm 5.12$ & $75.17 \pm 8.79$ & $56.45 \pm 3.32$ & N/A & N/A \\
Postinfarct & $42.86 \pm 4.37$ & $83.36 \pm 8.41$ & $48.23 \pm 3.29$ & $3.57 \pm 0.23$ & $0.25 \pm 0.01$ \\
2 weeks & $48.82 \pm 2.73^{*}$ & $85.79 \pm 3.92$ & $43.17 \pm 1.30^{*}$ & $4.79 \pm 0.36 \dagger$ & $0.31 \pm 0.02 \dagger$ \\
6 weeks & $62.43 \pm 3.41^{*}$ & $95.11 \pm 7.69$ & $39.97 \pm 2.06^{*}$ & $5.59 \pm 0.48 \dagger$ & $0.37 \pm 0.04 \dagger$ \\
Terminal & $74.33 \pm 10.63^{*}$ & $112.65 \pm 12.63^{*}$ & $37.07 \pm 3.35^{*}$ & $7.28 \pm 0.87 \dagger$ & $0.44 \pm 0.04 \dagger$
\end{tabular}

Summary of changes observed in left ventricular end-systolic volume (LVESV), left ventricular end-diastolic volume (LVEDV), ejection fraction (EF), wall motion abnormality (WMA), and ratio of wall motion abnormality to circumference (WMA/Circ). N/A, Not applicable. All values are given as mean \pm standard error of the mean. $* P \leq .05$ as compared with preinfarct. $\dagger P \leq .05$ as compared with postinfarct.

assess regional myocardial contractile function. The crystal positions at end-diastole were used as the reference configuration (frame), and subsequently the crystal positions were used as the deformed configuration. The strains between end-diastole and end-systole are commonly used to assess the LV regional systolic function. Therefore, the negative strain during LV systole indicates functional contraction of myocardium. Dysfunctional or stretched myocardium may appear to have positive systolic strain.

The end-diastolic (remodeling) strain was calculated by use of the LV free wall deformation from the initial pre-MI geometry during the study period after the MI. Using crystal positions at end-diastole before MI and at subsequent times after MI, we compared the deformed configurations to the pre-MI reference configuration to assess the regional change (such as, regional area expansion) resulting from LV remodeling.

The criteria for exclusion were (1) if the transducer did not maintain adequate transmission for the entire duration of study and/or (2) if the transducer became dislodged so as not to lie in the reconstructed 3-dimensional contour of the LV free wall.

\section{Histology}

At the time of the terminal study, the excised heart from each animal was harvested in ice-cold solution with tissue sections taken from the 3 zones of interest; infarct, adjacent (defined as $\leq$ $2 \mathrm{~cm}$ from edge of infarct), and remote zones of the LV free wall. The tissue was examined in paraffin-embedded sections of 5-mm cuts and stained with hematoxylin and eosin as well as Masson's trichrome stain.

\section{Regional Myocardial Perfusion}

Serial 3-mL injections of NuFlow fluorescent microspheres (5 million spheres/mL, 15.5- $\mu \mathrm{m}$ diameter, color-coded) (IMT Laboratories, Irvine, Calif) were carried out at baseline, after MI, and at the time of terminal study using the left atrial port catheter. Reference blood samples were taken from the right femoral artery with a constant-withdrawal syringe (Harvard Apparatus Co, Cambridge, Mass) beginning 5 seconds before microsphere injection at a constant rate of $15 \mathrm{~mL} / \mathrm{min}$ for a total of 80 seconds.

After the terminal study, myocardial tissue samples from the excised heart were taken with correlation to the ischemic myocardial borders at initial instrumentation. The tissues were separated into the infarct region, the adjacent region (defined as $<2 \mathrm{~cm}$ from infarct border), and the remote region. Myocardial samples along with reference blood samples were sent to IMT Laboratories for determination of regional myocardial perfusion as described elsewhere. ${ }^{8}$

\section{Western Blot Analysis}

For protein expression analysis, tissue samples corresponding to different regions were collected, rapidly frozen in liquid nitrogen, and stored at $-80^{\circ} \mathrm{C}$. Frozen tissue samples were homogenized in radioimmunoprecipitation assay buffer $(0.05$ Tris- $\mathrm{HCl}, \mathrm{pH} 7.4$, $0.15 \mathrm{~mol} / \mathrm{L} \mathrm{NaCl}, 0.25 \%$ deoxycholic acid, $1 \% \mathrm{NP}-40,1 \mathrm{mmol} / \mathrm{L}$ ethylenediaminetetraacetic acid, $1 \mathrm{mmol} / \mathrm{L}$ phenylmethylsulfonyl fluoride, $1 \mathrm{mmol} / \mathrm{L}$ sodium orthovanadate, $1 \mathrm{mmol} / \mathrm{L}$ sodium fluoride, $1 \mu \mathrm{g} / \mathrm{mL}$ aprotinin, $1 \mu \mathrm{L} / \mathrm{mL}$ leupeptin, and $1 \mu \mathrm{L} / \mathrm{mL}$ pepstatin), separated by sodium dodecylsulfate-polyacrylamide gel electrophoresis, transferred to nitrocellulose polyvinylidene difluoride membrane, and probed with specific primary antibodies. Protein loading was controlled by probing for glyceraldehyde-3phosphate dehydrogenase pseudogene (Santa Cruz Biotechnology, Santa Cruz, Calif). Expressions of sarcoplasmic reticulum $\mathrm{Ca}^{2+}$ adenosine triphosphatase subtype 2a (SERCA2a) at $110 \mathrm{kD}(1$ : 2000 dilution; Novocastra, Newcastle, United Kingdom), sodium/ calcium exchanger type $1(\mathrm{NCX}-1)$ cumulative at $120 \mathrm{kD}$ and 70 $\mathrm{kD}$ (1:500 dilution; Abcam, Cambridge, Mass), and phospholamban (PLB) cumulative at $25 \mathrm{kD}$ and $5 \mathrm{kD}$ (1:2000 dilution; Afinity BioReagents, Golden, Colo) were digitized and quantified with software (Silk Scientific, Orem, Utah; UN-SCAN-IT gel TM 5.1) that is sensitive to Western blot development by Enhanced Chemiluminescence Plus (ECL PLUS; Amersham, Piscataway, NJ).

\section{Statistical Analysis}

Data are given as mean \pm standard error of the mean. Comparisons between baseline and measurements at subsequent times were carried out by analysis of variance while comparisons between protein expressions were carried out with a Student paired $t$ test. Regression analyses with correlation coefficients are as shown.

\section{Results}

\section{Hemodynamics}

During the course of the study period, the heart rate, mean arterial pressure, and cardiac output remained relatively preserved without any statistically significant changes. The LV end-diastolic pressure, however, increased incrementally during the study period. More specifically, the pres- 


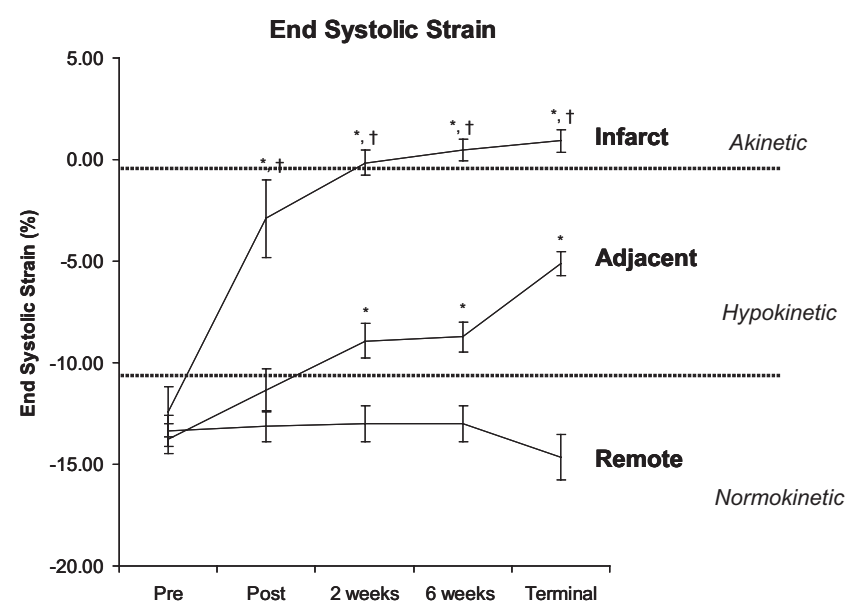

Figure 1. Regional end-systolic strain over time. The infarct region shows loss of function immediately post-MI and shows paradoxical dilatation by 2 weeks post-MI. The adjacent region shows a statistically significant systolic dysfunction by 2 weeks post-MI with rapid progression after 6 weeks post-MI. The remote region shows preserved systolic function and a nonstatistically significant trend toward hypercontractility by the time of terminal study. All values are given as mean \pm standard error of the mean. ${ }^{*} \boldsymbol{P} \leq .05$ as compared with remote region at that time point. $+P \leq .05$ as compared with adjacent region at that time point.

sures were $1.4 \pm 0.4,1.4 \pm 0.3,3.9 \pm 0.7(P \leq .05), 6.9$ $\pm 0.7(P \leq .05)$, and $8.1 \pm 0.8 \mathrm{~mm} \mathrm{Hg}(P \leq .05)$ at baseline, immediately post-MI, 2 weeks post-MI, 6 weeks post-MI, and at the time of terminal study. This observation is consistent with previous literature in showing an increase in LV end-diastolic pressure during cardiac remodeling and heart failure. 9

\section{Echocardiogram}

Echocardiographic data from the various study time points are summarized in Table 1 . The results from the measurement of the LV volume and global LV function showed increases in LV end-systolic volume from $33.54 \pm 5.12 \mathrm{~mL}$ to $74.33 \pm 10.63 \mathrm{~mL}(P \leq .05)$ and end-diastolic volume from $75.17 \pm 8.79 \mathrm{~mL}$ to $112.65 \pm 12.63 \mathrm{~mL}(P \leq 0.05)$ with a resultant decrease in ejection fraction from $56.45 \% \pm$ $3.32 \%$ to $37.07 \% \pm 3.35 \%(P \leq .05)$ during the progression of $\mathrm{LV}$ remodeling. Based on analysis of the short-axis echocardiogram around papillary muscle, infarct expansion was observed with an increase in the length of wall motion abnormality from $3.57 \pm 0.23 \mathrm{~cm}$ immediately post-MI to $7.28 \pm 0.87 \mathrm{~cm}(P \leq .05)$ at the time of terminal study. Likewise, the ratio of wall motion abnormality to endocardial circumference increased from $0.25 \pm 0.01$ to $0.44 \pm$ $0.04(P \leq .05)$, illustrating the more rapid expansion of

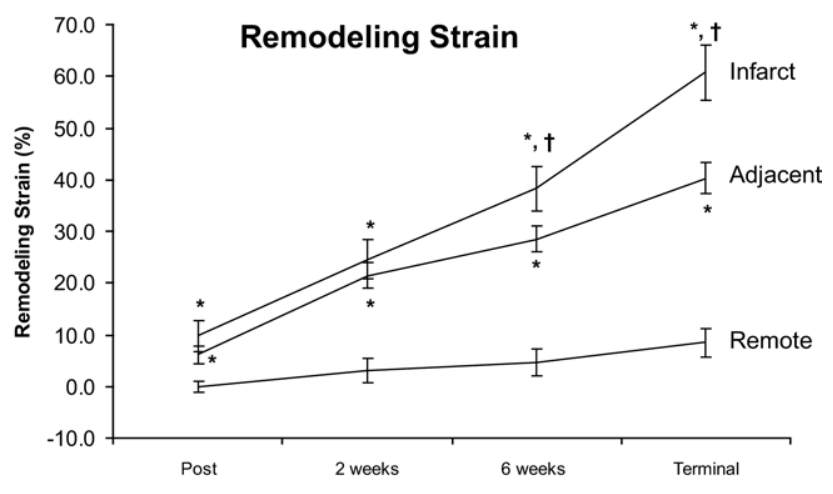

Figure 2. Regional end-remodeling strain over time. The infarction region consistently shows the greatest remodeling strain while the adjacent region undergoes a similar remodeling strain, although to a lesser degree than the infarct region. Of note, the greatest increase in end-diastolic strain is observed between 6 weeks post-MI and the time of terminal study. All values are given as mean \pm standard error of the mean. ${ }^{*} P \leq .05$ as compared with remote region at that time point. $+P \leq .05$ as compared with adjacent region at that time point.

infarct tissue as compared with the adjacent and remote myocardium.

\section{Strain}

Serial regional end-systolic strain data from a representative animal over the duration of the study are shown in Figure 1. Negative strain values (expressed as percent change over diastolic reference) designate the functional shortening (contraction) and positive values indicate lengthening (dilatation). At baseline, all 3 zones are noted to have relatively normal strain waveforms with end-systolic strain values being measured as $-12.42 \% \pm 1.23 \%,-13.75 \% \pm 0.74 \%$, and $-13.37 \% \pm 0.76 \%$ in the infarct, adjacent, and remote zones, respectively. Immediately after MI, however, the infarct zone lost contractile function whereas the adjacent and remote zones remained unchanged. Over the duration of the study, the systolic strain in the infarct zone approached zero, illustrating akinesis. The adjacent and remote zones also exhibited different trends in systolic strain. The adjacent zone became hypokinetic, with gradual decrease of strain from $-13.75 \%$ to $-6.0 \%$, whereas the remote zone remained statistically unchanged.

The end-diastolic (remodeling) strain at 4 time points is illustrated in Figure 2. Both the infarct and adjacent zones had a progressive expansion immediately after MI with the largest percentage of increase between 6 weeks post-MI and the terminal study, regardless of the tissue region. The remote zone had a much smaller remodeling strain than did the other 2 zones. 

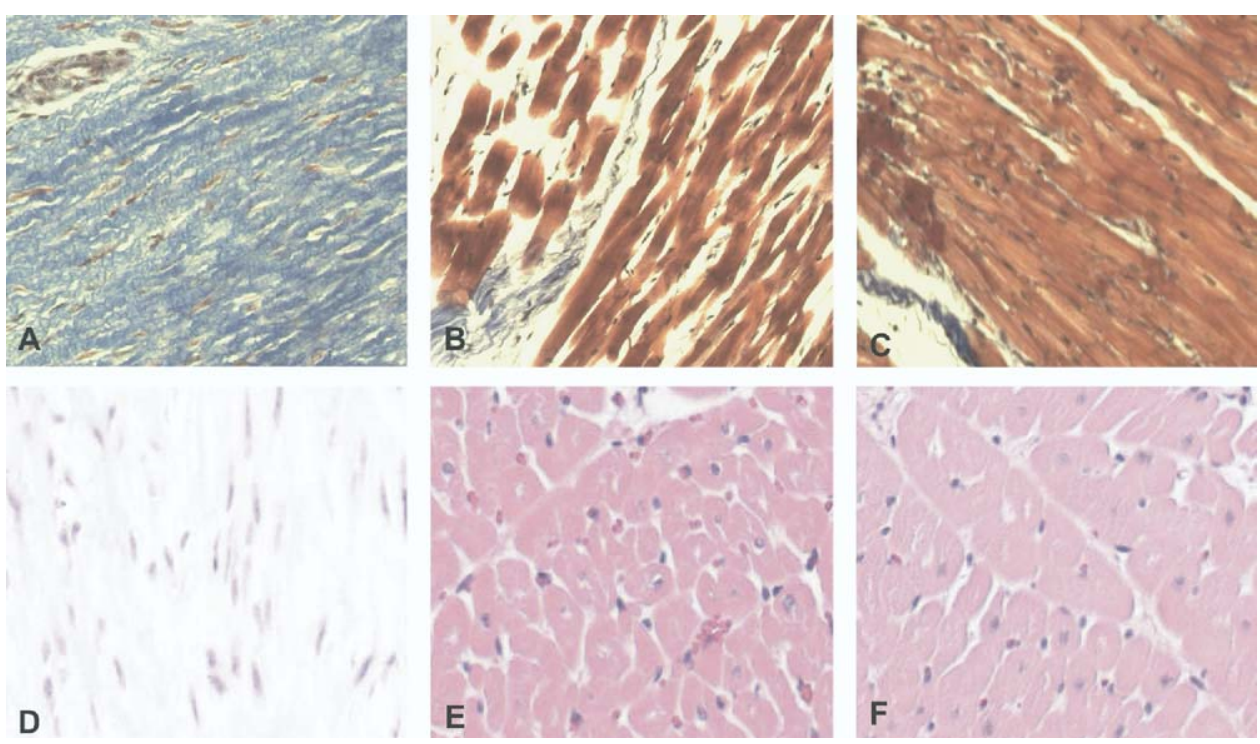

Figure 3. Histologic sections of myocardium from the infarct $(A$, $D)$, adjacent $(B, E)$ and remote $(C, F)$ zones examined with Masson's trichrome (top) and hematoxylin and eosin (bottom) staining (magnification, $\times 20$ ). The infarct zone shows marked fibrosis and collagen deposition. The adjacent zone shows lesser degree of fibrosis with myocyte vacuolization visible on hematoxylin-eosin stain. The remote zone has normal, well-preserved myocyte morphology.

\section{Histology}

Histologic sections of the 3 different myocardial regions with hematoxylin and eosin as well as Masson's trichrome stain are shown in Figure 3. The infarct region showed fibrosis consistent with an 8- to 12-week-old MI. The adjacent region showed mild hypertrophy with fibrosis at the infarct edge. The remote region demonstrated preserved normal myocardium.

\section{Regional Myocardial Perfusion}

The regional myocardial blood flow in the nonischemic zones (adjacent and remote) remained constant throughout the study period. The remote zone regional myocardial perfusions at baseline, post-MI, and terminal study were $1.09 \pm 0.07,1.37 \pm 0.11$, and $1.26 \pm 0.12 \mathrm{~mL} \cdot \mathrm{min}^{-1} \cdot \mathrm{g}^{-1}$ of tissue, whereas the adjacent zone myocardial perfusions at the same time periods were $1.05 \pm 0.09,1.12 \pm 0.13$, and $1.07 \pm 0.19 \mathrm{~mL} \cdot \min ^{-1} \cdot \mathrm{g}^{-1}$ of tissue. In contrast, the infarct regional blood flows were $1.19 \pm 0.15,0.07 \pm 0.02$ $(P \leq .05)$ and $0.53 \pm 0.18(P \leq .05)$.

\section{Calcium-handling Protein Expression}

The abundance of the major calcium regulatory proteins, SERCA2a, PLB, and NCX-1, in the infarct, adjacent, and remote zones is shown in Figure 4. The infarct region showed an 18-fold decrease in expression of SERCA2a and a 4-fold decrease in expression of PLB as compared with both the remote region and the tissues from uninstrumented, healthy animals. Although the infarct region showed the greatest alteration in protein expression from normal, the remote region showed preservation of protein function.

\section{Protein Expression as a Function of Strain}

The plots of SERCA2a, PLB, and NCX-1 protein expression versus end-systolic strain are shown in Figure 5. A negative relationship is observed for expression of SERCA2a $\left(r^{2}=0.68, P \leq .05\right)$ and PLB $\left(r^{2}=0.50, P \leq\right.$ $.05)$ with regard to end-systolic strain, illustrating that the more contractile the myocardium (ie, a more negative number), the higher the expression of SERCA2a and PLB. In contrast, NCX-1 expression has a weaker, but positive, correlation with end-systolic strain $\left(r^{2}=0.17, P \leq .05\right)$.

\section{Discussion}

Numerous studies on calcium handling have shown that myocytes possess mechanosensitivity ${ }^{10}$; that is, they are able to use stretch (strain) activated channels in changing calcium homeostasis. A strong relationship between stretch and calcium regulation dysfunction in decompensated cardiac remodeling has been reported. ${ }^{11}$ This alteration of calcium regulation with subsequent loss of excitationcontraction coupling has been implicated as playing a central role in both systolic and diastolic contractile dysfunction. ${ }^{12}$ In addition to affecting an individual myocyte, mechanically distorting one cell has been shown to raise the concentration of intracellular calcium in a neighboring cell. ${ }^{13}$ On a more regional level, numerous studies have shown dysregulation of proteins and genes involved in regional calcium handling. ${ }^{3,14-17}$ Recently, the clinical relevance of this mechanoelectric feedback has been demonstrated in patients undergoing cardiopulmonary bypass. During weaning from cardiopulmonary support, an increase in force and volume leads to a decrease in intracellular calcium and consequently decreased action potential dura- 
A

SERCA2a

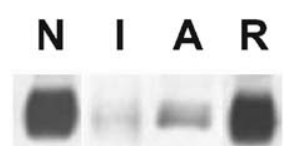

110 kD

NCX -1

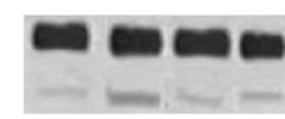

120 kD

$70 \mathrm{kD}$

PLB

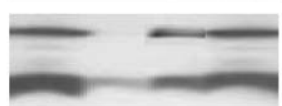

$25 \mathrm{kD}$

$5 \mathrm{kD}$

GAPDH

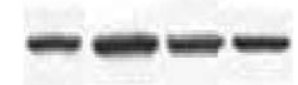

$37 \mathrm{kD}$

B

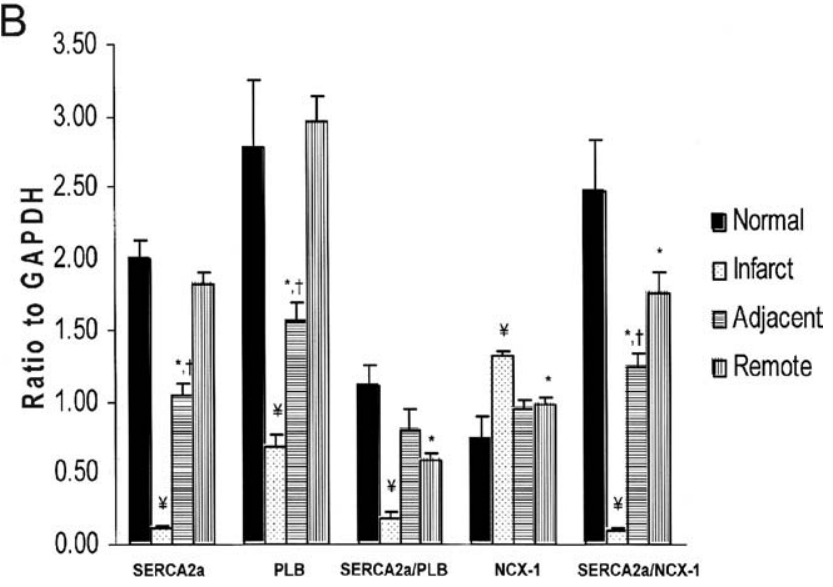

Figure 4. A, Representative Western blots. SERCA2a, Sarcoplasmic reticulum calcium adenosine triphosphatase type 2a; PLB, phospholamban; NCX-1, sodium/calcium exchanger type 1; GAPDH, glyceraldehyde-3-phosphate dehydrogenase; $N$, normal, reference animal; $I$, infarct zone in $\mathrm{MI}$ animal; $A$, adjacent zone in MI animal; $R$, remote zone in MI animal. B, Arbitrary density ratio to GAPDH. All values are given as mean \pm standard error of the mean. $¥ \boldsymbol{P} \leq .05$ as compared with normal, adjacent, and remote tissue. ${ }^{*} P \leq .05$ as compared with normal ovine tissue. $\dagger P \leq .05$ as compared with remote tissue.

tion, illustrating how stretch (strain) can affect excitationcontraction coupling via its effects on calcium. ${ }^{18}$

Both contractile strain ${ }^{16,19-22}$ and expression of calciumhandling proteins ${ }^{3,12,14-17,23-25}$ in LV myocardium have been studied extensively. In our present study, we investigate the relationship of both the systolic strain (contractile function) and remodeling strain (myocardial structure deformation) to alterations in protein expression during cardiac remodeling. Our experimental purpose was 2-fold. Our results show that there are regional strain differences in the infarct, adjacent, and remote zones of the post-MI myocardium. These regional strains correlate with expression of calcium-handling proteins of the remodeled myocardium as the end product of cardiac remodeling.
We have shown that regional differences in the systolic function of the post-MI myocardium follow a predictably progressive pattern. The end-diastolic (remodeling) changes were consistent with the in vivo changes observed in LV end-diastolic pressure and echocardiogram confirmed changes in LV end-diastolic volume, ejection fraction, and wall motion abnormality. Interestingly, the largest change in volumes, ejection fraction, and remodeling strain occurred at the period between 6 weeks post-MI and the time of terminal study. This was also the time period when the reduction in the end-systolic function was the greatest for the adjacent zone myocardium. As a consequence, the remote end-systolic strain trended toward hypercontractility. The compensatory hypercontractility of the remote zone has previously been shown in an acute ischemia model and has been directly correlated with mortality. ${ }^{24}$ Our findings in a long-term setting suggest that the critical period of decompensation after post-MI remodeling in the ovine model is from 6 to 8 weeks after the initial ischemic event. A possible mechanism for this period of rapid change is stiffening of the infarct tissue with resultant strain alterations in the adjacent myocardium leading to loss of function and hypocontractility.

In addition to showing temporal regional strain gradients among the 3 aforementioned zones, we have correlated local strain data with site-specific expression of calcium-handling proteins. Cardiac muscle and protein expression exhibit sensitivity toward being structurally stretched (strained) and there exists a feedback mechanism for excitationcontraction coupling. We believe that strain through mechanotransduction is a continuous impetus for the molecular changes observed in cardiac remodeling. Changes in myocardial remodeling strain can be seen immediately after MI not only on a global level but also on a more localized, regional level. The stretch (strain) serves as the local force from which local alterations in protein expression and cardiac function take place.

The potential clinical significance of this study relates to the measurement of this driving force with the hopes of preserving post-MI regional myocardial function. With advancements in technology, such as the 3-dimensional echocardiogram, regional strain can now be measured in a noninvasive manner.

It is our hope that in focusing on regional strain, a strain level can be established that will predict when post-MI myocardial remodeling with progression to heart failure is more likely to occur. The obvious clinical implication of this strain measurement is to take early steps toward preventing the development of heart failure. It has been shown that reduction of strain through ventricular endocardial restoration, ${ }^{25}$ passive constraint devices, ${ }^{26,27}$ and $\mathrm{LV}$ assist devices $^{28,29}$ can reverse remodeling with a near normalization of cardiac function and protein expression. Our prelim- 
inary studies with a post-MI LV assist device-supported animal group (data not shown) have reduced regional remodeling strain with resultant near normalization of the calcium-handling protein expression. Translation of these findings to clinical post-MI healing is largely dependent on the cadence of micro-ventricular assist device and passive
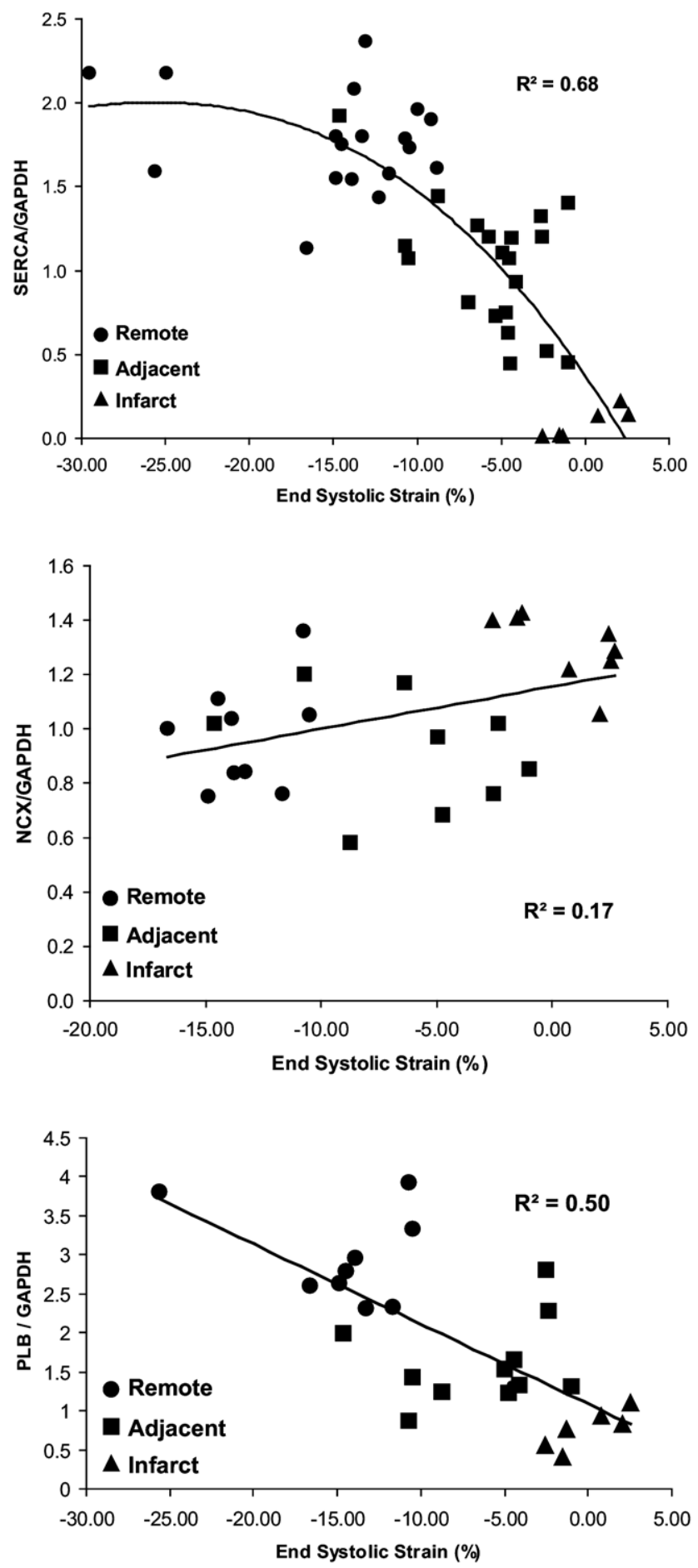

constraint device development. Regardless of the treatment chosen for post-MI hearts progressing toward heart failure, the role that strain plays in both healthy and diseased myocardium needs to be more clearly defined.

\section{References}

1. Anversa P, Loud AV, Levicky V, Guideri G. Left ventricular failure induced by myocardial infarction. I. Myocyte hypertrophy. Am J Physiol. 1985;248:H876-82.

2. Cohn JN, Ferrari R, Sharpe N. Cardiac remodeling-concepts and clinical implications: a consensus paper from an International Forum on Cardiac Remodeling. J Am Coll Cardiol. 2000;35:569-82.

3. Kim YK, Kim SJ, Kramer CM, Yatani A, Takagi G, Mankad S, et al. Altered excitation-contraction coupling in myocytes from remodeled myocardium after chronic myocardial infarction. J Mol Cell Cardiol. 2002:34:63-73.

4. Wilson EM, Moainie SL, Baskin JM, Lowry AS, Deschamps AM, Mukherjee R, et al. Region- and type-specific induction of matrix metalloproteinases in post-myocardial infarction remodeling. Circulation. 2003;107:2857-63.

5. Ashikaga H, Mickelsen SR, Ennis DB, Rodriguez I, Kellman P, Wen $\mathrm{H}$, et al. Electromechanical analysis of infarct border zone in chronic myocardial infarction. Am J Physiol Heart Circ Physiol. 2005;289: H1099-105.

6. Wyatt HL, Meerbaum S, Heng MK, Gueret P, Corday E. Crosssectional echocardiography. III. Analysis of mathematic models for quantifying volume of symmetric and asymmetric left ventricles. $A m$ Heart J. 1980;100:821-8.

7. Holmes JW, Yamashita H, Waldman LK, Covell JW. Scar remodeling and transmural deformation after infarction in the pig. Circulation. 1994;90:411-20.

8. Takayama Y, Holmes JW, LeGrice I, Covell JW. Enhanced regional deformation at the anterior papillary muscle insertion site after chordal transsection. Circulation. 1996;93:585-93.

9. Moainie SL, Gorman JH 3rd, Guy TS, Bowen FW, Jackson BM Plappert T, et al. An ovine model of postinfarction dilated cardiomyopathy. Ann Thorac Surg. 2002;74:753-60.

10. Lab MJ. Mechanoelectric feedback (transduction) in heart: concepts and implications. Cardiovasc Res. 1996;32:3-14.

11. Calaghan SC, White E. The role of calcium in the response of cardiac muscle to stretch. Prog Biophys Mol Biol. 1999;71:59-90.

12. Gómez AM, Valdivia HH, Cheng H, Lederer MR, Santana LF, Cannell MB, et al. Defective excitation-contraction coupling in experimental cardiac hypertrophy and heart failure. Science. 1997; 276:800-6

13. Sigurdson W, Ruknudin A, Sachs F. Calcium imaging of mechanically induced fluxes in tissue-cultured chick heart: role of stretch-activated ion channels. Am J Physiol. 1992;262(4 Pt 2):H1110-5.

14. Heerdt PM, Holmes JW, Cai B, Babone A, Madigan JD, Reiken S, et al. Chronic unloading by left ventricular assist device reverses con-

\footnotetext{
Figure 5. Normalized sarcoplasmic reticulum calcium adenosine triphosphatase type 2a (SERCA2a), phospholamban (PLB), and sodium/calcium exchanger type 1 (NCX-1) to glyceraldehyde-3phosphate dehydrogenase (GAPDH) plotted as a function of endsystolic strain. A strong negative relationship between SERCA2a (top) and PLB (middle) expression versus end-systolic strain can be observed. A modest positive relationship between NCX-1 (bottom) and end-systolic strain is also observed. At end-systole, the positive strain values indicate lengthening (dilatation) and are seen in the infarct region. Negative strain values correspond with shortening (contraction) and are seen in the adjacent and remote zones.
} 
tractile dysfunction and alters gene expression in end-stage heart failure. Circulation. 2000;102:2713-9.

15. Chaudhary KW, Rossman EI, Piacentino V 3rd, Kenessey A, Weber C, Gaughan JP, et al. Altered myocardial $\mathrm{Ca}^{2+}$ cycling after left ventricular assist device support in the failing human heart. J Am Coll Cardiol. 2004;44:837-45.

16. Ito K, Yan X, Tajima M, Su Z, Barry WH, Lorell BH. Contractile reserve and intracellular calcium regulation in mouse myocytes from normal and hypertrophied failing hearts. Circ Res. 2000;87:588-95.

17. Kim SJ, Kudej RK, Yatani A, Kim YK, Takagi G, Honda R, et al. A novel mechanism for myocardial stunning involving impaired $\mathrm{Ca}^{2+}$ handling. Circ Res. 2001;89:831-7.

18. Taggart P, Sutton PMI. Cardiac mechano-electric feedback in man: clinical relevance. Prog Biophys Mol Biol. 1999;71:139-54.

19. Yeon SB, Reichek N, Tallant BA, Lima JA, Calhoun LP, Clark NR, et al. Validation of in vivo myocardial strain measurement by magnetic resonance tagging with sonomicrometry. J Am Coll Cardiol. 2001;38: 555-61.

20. Smiseth OA, Ihlen H. Strain rate imaging: why do we need it? $J$ Am Coll Cardiol. 2003;42:1584-6.

21. Hashimoto I, Li X, Bhat AH, Jones M, Zetts AD, Sahn DJ. Myocardial strain rate is a superior method for evaluation of left ventricular subendocardial function compared with tissue doppler imaging. $J \mathrm{Am}$ Coll Cardiol. 2003;42:1574-83.

22. Bers DM, Cardiac excitation-contraction coupling. Nature. 2002;415: 198-205.

23. Yano M, Ikeda Y, Matsuzaki M. Altered intracellular $\mathrm{Ca}^{2+}$ handling in heart failure.J Clin Invest. 2005;115:556-64.

24. Beyersdof F, Acar C, Buckberg GD, Partington MT, Sjostrand F, Young $\mathrm{HH}$, et al. Studies on prolonged acute regional ischemia. III. Early natural history of simulated single and multivessel disease with emphasis on remote myocardium. J Thorac Cardiovasc Surg. 1989; 98:368-80.

25. Athanasuleas CL, Stanley AWH Jr, Buckberg GD, Dor V, Di Donato M, Blackstone EH, et al. Surgical anterior ventricular endocardial restoration (SAVER) in the dilated remodeled ventricle after anterior myocardial infarction. J Am Coll Cardiol. 2001;37:1199-209.

26. Blom AS, Mukherjee R, Pilla JJ, Lowry AS, Yarbrough WM, Mingoia JT, et al. Cardiac support device modifies left ventricular geometry and myocardial structure after myocardial infarciton. Circulation. 2005; 112:1274-83.

27. Sabbah HN, Sharov VG, Gupta RC, Mishra S, Rastogi S, Undrovinas AI, et al. Reversal of chronic molecular and cellular abnormalities due to heart failure by passive mechanical ventricular containment. Circ Res. 2003;93:1095-101.

28. Razeghi P, Myers TJ, Frazier OH, Taegtmeer H. Reverse remodeling of the failing human heart with mechanical unloading. Cardiology. 2002;98:167-74.

29. Müller J, Wallukat G, Weng YG, Dandel M, Speigelsberger S, Semrau $\mathrm{S}$, et al. Weaning from mechanical cardiac support in patients with idiopathic dilated cardiomyopathy. Circulation. 1997;96:542-9.

\section{Discussion}

Dr John V. Conte (Baltimore, $M d$ ). We all know that post-MI remodeling is a very complex process that occurs at microscopic and macroscopic levels, and it is hard to differentiate what happens at what time period.

You have very nicely demonstrated changes in regional strain and changes in protein expression. Is this a cause-and-effect relationship, or are these events that are true, true and unrelated? How do we unify these 2 processes? I think the concept of mechanotransduction certainly is appealing, but how have we shown that it, in fact, is occurring?

Second, you have demonstrated beautifully that regional strain affects the calcium-handling proteins. Are any other proteins affected that you know of? If there are, that would tend to support your hypothesis that, in fact, it is mechanotransduction that has caused these changes.
Third, what we have seen demonstrated here in this model is the natural history of an MI. We know that that is not how MIs are treated today. If you had added beta-blockers and angiotensinconverting enzyme inhibitors to this model, do you think you would have had the same results as you have demonstrated?

Passive restraint devices have been shown to reduce and in some cases prevent post-MI remodeling. In this model, post-MI remodeling occurred unabated. If you had put on passive restraint devices so that the post-MI dilation did not develop, do you have any evidence that you would still have these changes in protein expression?

Finally, you mentioned that there is a time period between 6 weeks and the end of the study that these changes in strain were most profound. If we as surgeons are going to intervene, and that would mean restraint devices or even ventricular assist devices to decrease the wall stress, at what time period should we intervene? Have you shown us the ideal window for us to intervene, or is this just an effect that happened based on the length of time of your study? If your study had continued for a longer period of time, do you think we might find out that there is another time period where these effects could become even more pronounced and we should wait for those?

Dr Kilic. I can answer your first 2 questions regarding the cause-and-effect relationship and other proteins-of-interest analysis by saying that we have investigated the role of stretch-activated $\mathrm{G}$ protein-coupled receptors and the phosphoinositide-3-kinases. Classically, these families of proteins are associated with stretchinduced activation of apoptosis, hyperhypertophy, and contractility. In our experiments, we see the same trends of strain-graded response in the adjacent and remote regions.

In terms of the beta-blockade and angiotensin-converting enzyme inhibitors, we showed that in the remote region both strain and protein expression are preserved, whereas in the infarct region, the protein expression becomes completely dysfunctional with akinetic strain. I think if the medications (beta-blockers and angiotensin-converting enzyme inhibitors) were to make a difference, they would have mitigated the degree of strain loss and the degree of protein loss in the adjacent region.

With regard to your question about the constraint device, we have some experience with strain offloading using a small ventricular assist device in our model. In addition to the obvious reduction in strain loss, we actually observe a near normalization of protein expression in the adjacent region. In using the ventricular assist device, we also reduce the size of the infarct and preserve overall myocardial contractility. Our experience with the small ventricular assist device would likely be comparable to the use of constraint devices, but to what degree I cannot elaborate.

Your last question regarding the window of opportunity for intervention is an interesting one. We do see an overall decompensation that occurs between 6 weeks and the time of terminal study (at 8 to 10 weeks). This is largely due to the fact that our model is intended to induce heart failure at around that time period. So in terms of the best time to intervene, that is a question that we would hope to answer in our future studies. However, I think the ideal time will be before the adjacent region becomes decompensated and the remote region becomes hypercontractile. This would mean definitely before 6 weeks, but how early immediately after MI I cannot comment. 
Dr Bruno K. Podesser (Vienna, Austria). Did you really expect to have a lot of calcium-handling proteins in the scar left? Normally you will find mainly fibroblasts there and very few myocytes.

The second thing I wanted to point out is that I think extracellular matrix remodeling proteins, like the tissue inhibitors of metalloproteinases or matrix metalloproteinases, might have influenced your results even more. Could you comment on that?

Dr Kilic. Your question is very valid. How can we compare scar tissue to myocytes-basically infarct tissue with adjacent/ remote tissue?

We do have other data where we have shown with Bax, other proteins involved in apoptosis (Akt/BAD), and matrix metalloproteinases where there is actually an increase in protein expression that occurs in the infarct region.

In addition, I would point out that our protein expression for the sodium-calcium exchanger in the infarct tissue actually has increased. So, in fact, there is a degree of protein expression in the scar tissue. How pertinent is protein expression in the infarct region? You can see a clear relationship between strain and protein expression even without the infarct tissue when you look at the differences between just the adjacent and remote regions. There exists a gradient of strain and protein expression loss as we near the infarct region.

Dr Pedro Catarino (London, United Kingdom). One would expect some degree of hypertrophy even at this early stage as part of the remodeling process.

First, how do you normalize your protein expression for the hypertrophy?

Second, do you have a view on whether your protein expression changes reflect more myocyte hypertrophy or myocyte failure?

Dr Kilic. In terms of myocyte hypertrophy, we did take histologic sections from the adjacent and remote regions as well as the infarct region. What we observed was that the adjacent region does show relatively more hypertrophy than the remote regions. That being said, the hypertrophy does not seem to be directly causing an overexpression of protein. In fact, the adjacent region is where we see a loss of protein expression. Although we have not looked at the ratio of myocyte size to protein expression, I do not believe there would be an up-regulation resulting from hypertrophy. 